\title{
An Innovative Prostate Cancer Detection Method via Model Checking
}

\author{
Antonella Santone \\ University of Molise \\ Francesco Mercaldo ( $\nabla$ francesco.mercaldo@unimol.it ) \\ University of Molise \\ Maria Chiara Brunese \\ University of Molise \\ Federico Donnarumma \\ University of Molise \\ Pasquale Guerriero \\ University of Molise \\ Alfonso Reginelli \\ University of Campania "Luigi Vanvitelli"
}

\section{Research Article}

Keywords: Cancer, Medicine, ROI

Posted Date: June 4th, 2021

DOI: https://doi.org/10.21203/rs.3.rs-579730/v1

License: (c) (i) This work is licensed under a Creative Commons Attribution 4.0 International License.

Read Full License 


\title{
An Innovative Prostate Cancer Detection Method via Model Checking
}

\author{
Antonella Santone ${ }^{1, *,+}$, Francesco Mercaldo ${ }^{1, *,+}$, Maria Chiara Brunese ${ }^{1,+}$, Federico \\ Donnarumma ${ }^{1,+}$, Pasquale Guerriero ${ }^{1,+}$, and Alfonso Reginelli ${ }^{2,+}$
}

${ }^{1}$ Department of Medicine and Health Sciences "Vincenzo Tiberio", University of Molise, Campobasso, Italy

${ }^{2}$ Department of Precision Medicine, University of Campania "Luigi Vanvitelli", Napoli, Italy

* $\{$ antonella.santone, francesco.mercaldo\}@unimol.it

+these authors contributed equally to this work

\begin{abstract}
Purpose: one of typical cancer among men is the prostate tumour. This is the reason why the screening and the early detection is a crucial task to obtain a diagnosis and a subsequent therapy in the shortest possible time. Materials and Methods: in this paper, with the aim to help radiologists and pathologists for a prompt diagnosis, a method for detecting prostate cancer is proposed. Our analysis starts from the magnetic resonances images (coronal and axial planes), building a labelled transition system for coronal slices and another one for axial, which takes into account a number of non invasive radiomic features. Thus, a set of formulae in temporal logic characterizing the prostate cancer is verified through the model checking technique, to detect the prostate cancer. The proposed method considers magnetic resonance images without the Region Of Interest. This represents one of the major novelty of the method. Results: the proposed method is evaluated on a data-set composed of 40 patients, obtaining very interesting performances in the discrimination between affected and not affected prostate cancer patients. Conclusion: the study confirms the effectiveness of the formal methods to discriminate between cancerous and benign prostate MRIs with a method not requiring the ROI of the cancerous area, by obtaining a sensitivity and a specificity equal to 1.
\end{abstract}

\section{Introduction}

The tumour of the prostate cancer represents one of the most common cause of cancer death among men worldwide ${ }^{1}$ and the second and third leading cause of cancer-related death in the United States and Europe, respectively ${ }^{2,3}$. It has a strong tropism towards prostate tissue of adult males, about 2 tumors on 3 belong to over 65 years old men. Moreover, among the main risk factors for prostate cancer, familiarity must be considered, but also variability gene-related like anomalies in BRCA 1 and BRCA2 genes, already known for their relations with ovary and breast cancer, and the gene HPC1(https://www.airc.it/). These patients have more elevated possibilities to develop the cancer and they are suggested to attend screening programs and follow up to identify early the lesions or to monitor them.

In this context, the fine-grain detection of the suspicious prostate cancer $(\mathrm{PCa})$ is of great interest with the to manage in an appropriate way the disease.

The Prostate Imaging and Reporting Archiving Data System version 2 (PI-RADS v2) ${ }^{4}$ was introduced with the aim to propose a standard in MpMRI interpretation. It is a score, from 1 to 5, to quantify the grade of suspicious PCa.

Radiomics represents a new research field devoted to the extraction of numerical biomarkers from medical images ${ }^{5}$. Using these biomarkers it has been shown that it is possible to identify pathologies even at an early stage. Most exploited radiomics features consider volume, shape and size. Furthermore, considering that radiomic features are typically extracted from medical images, they represent a non-invasive technique ${ }^{6}$ for disease detection. Moreover they can be extracted from any organ. In the prostate cancer context, radiomics can be used to highlight the various lesions of the PI-RADS scale in a totally automatic but above all non-invasive way, thus avoiding the patient's biopsy.

In this paper, a method to detect whether a magnetic resonance image exhibits the prostate cancer is proposed. The underlying idea behind the proposed method is to offer to specialists the possibility to detect the PCa in an early stage i.e., when the patient has no symptoms. The possibility of identifying a cancer in a early stage, when is therefore small and not very extensive, allows doctors to treat with less invasive therapies, such as minor resection surgeries, and with better chances of success. Thus, the method can be used as screening for prostate cancer and as support for doctors. We exploit a set of radiomic features computed directly on the whole organ: the proposed method does not require to mark the MRIs with the Region Of Interest (ROI). This is an important and novel result, since we can see what the expert's eye does not see. A method using 
images with ROI, may not discover a cancer outside the ROI itself, if the ROI has not been correctly marked. Thus, in this work we consider the whole organ, without losing the analysis of potentially carcinogenic areas. Another important novelty is represented by the analysis considering two different representations of the organ related to the axial and coronal plane. This allows to obtain better performances in the pathology detection, since two orthogonal views of the same image are considered.

Our method basically considers MRIs in terms of formal models and through the model checking technique a series of temporal logic formulae (describing the prostate cancer) are verified by exploiting a formal verification environment.

Below, we itemize the distinctive points of the proposed method:

- we represent the patient (coronal and axial) slices in terms of labelled transition system built exploiting radiomic features;

- the proposed feature set is generated on the whole organ (this aspect represents one of the principal novelty of our proposal);

- a set of temporal logic formulae aimed to identify the prostate tumour in coronal and axial magnetic resonance images;

- an experiment aimed to assess the effectiveness of the proposed method on 40 different patient MRIs marked by pathologists as affected or not-affected by prostate cancer;

- from the experimental evaluation we reach a specificity and a sensitivity equal to 1 .

The paper proceeds as follows: the next section describe the proposed method, the results of the experimental evaluation are shown in Section 1, current state of the art literature is described in Section 1 and, finally, conclusion and future research direction are discussed in the last section.

\section{Method and Material}

In this section we describe our approach to detect prostate cancer by MRI analysis through model checking.

Figure 1 depicts the proposed methodology.

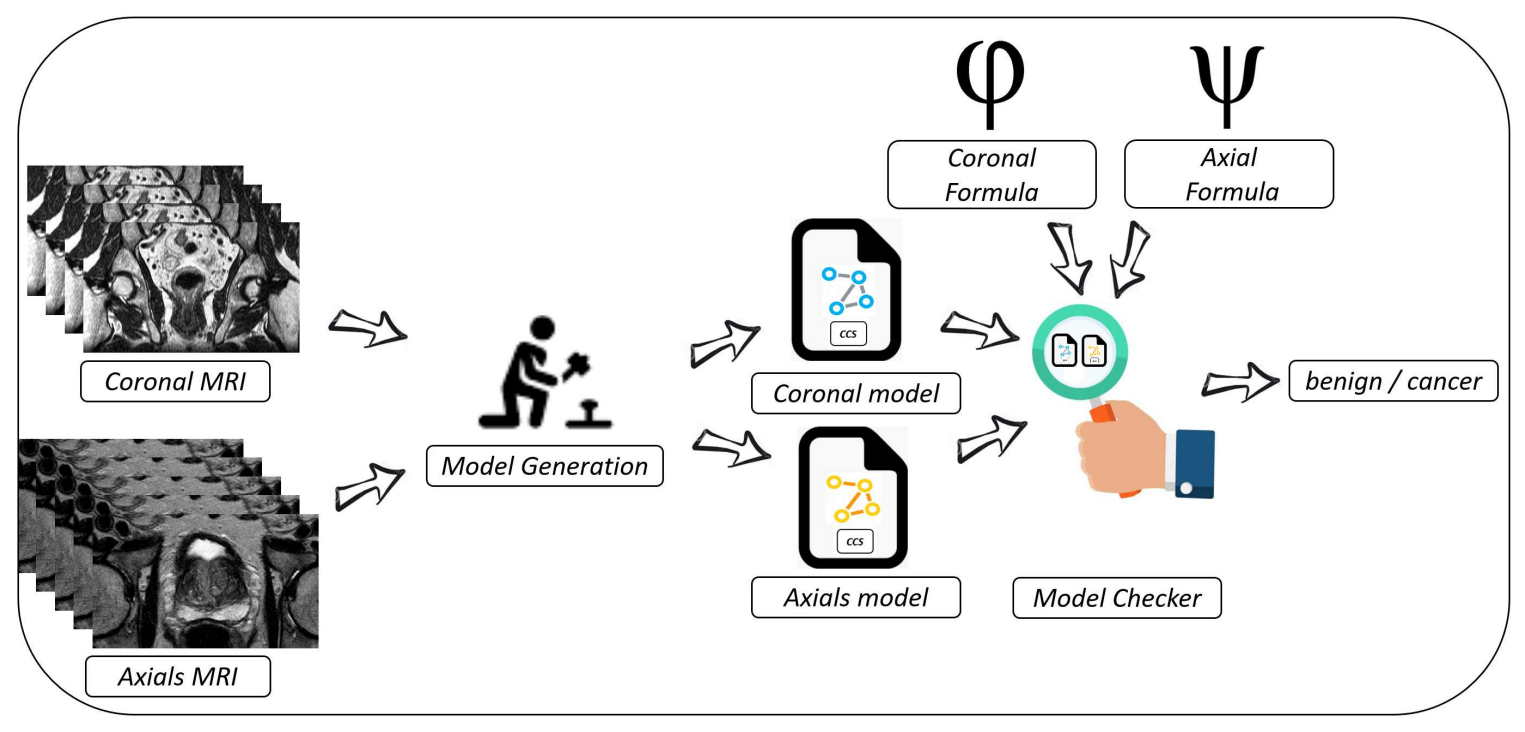

Figure 1. The method.

The analysis is starting from a set of coronal and axial MRIs. We take into account these two different representations of the same patient to have a complete view of the possible pathology and to define a robust method to effectively detect the cancer. The MR image formation process subdivides a section of the body of the patient into a set of slices: we consider, for each patient under analysis, the slices belonging to coronal and axial MRIs. From the set of coronal and axial slices, we compute a set of numeric values i.e., the shape (radiomic) features defined in Table 1.

The radiomic features are extracted on without-ROI slices and to gather radiomic features the pyradiomics package ${ }^{7}$ is invoked by using a software implemented by authors by exploiting the Python programming language. Subsequently, the numeric values of the radiomic features are discretised, i.e., we divide the numeric values into discrete ones. There are several algorithms proposed by community research for variable discretisation, in this paper we resort to the equal-width discretization ${ }^{8}$ : 
Table 1. Features involved in the study.

\begin{tabular}{|c|c|c|}
\hline Feature & Feature Name & Feature Description \\
\hline$R F 1$ & $\begin{array}{l}\text { Shape Maximum 2d Diameter } \\
\text { Slice }\end{array}$ & $\begin{array}{l}\text { The largest pairwise Euclidean distance between } \\
\text { the cancer surface mesh vertices in the row- } \\
\text { column plane. }\end{array}$ \\
\hline RF2 & Shape Least Axis Length & $\begin{array}{l}\text { This feature yields the second-largest axis length } \\
\text { of the ellipsoid. }\end{array}$ \\
\hline RF3 & Shape Major Axis Length & $\begin{array}{l}\text { This feature yields considers the largest axis } \\
\text { length of the ellipsoid and it is computed } \\
\text { exploiting the largest principal component. }\end{array}$ \\
\hline RF4 & $\begin{array}{l}\text { Shape Maximum 2d Diameter } \\
\text { Row }\end{array}$ & $\begin{array}{l}\text { It is defined as the largest pairwise Euclidean } \\
\text { distance between cancer surface mesh vertices } \\
\text { in the column-slice plane. }\end{array}$ \\
\hline
\end{tabular}

this algorithm first finds the minimum and the maximum values for each radiomic feature $R F_{x}$ with $1 \leq x \leq 4$, and then divides this range into a number $I_{R F_{x}}$ of equal-width intervals (this parameter is user-specified, in this work we consider $I_{R F_{x}}=3$ ).

Once obtained the discretised intervals for the radiomic features, we represent coronal and axial MRI in terms of Labeled Transition System i.e.,LTS, basically composed by nodes and edges aimed to connect the related nodes.

The LTS is composed by the possible interleaving of the $I_{R F_{x}}$ intervals for the $i$-th slice to compose the final model including all the patient slices. For each patient, two models are generated, the first one is related to the coronal slices while the second for the axial ones. Once obtained the formal models (i.e., the coronal and the axial models), we need to define logical temporal formulae aimed to prostate tumour identification (i.e., to characterize the cancer). We consider a precise notation provided by the temporal mu-calculus $\operatorname{logic}^{9}$ that allow us to state in a formal way the prostate cancer characterization. It is important to use a temporal logic to express how the features change in the slice sequence.

To check whether the temporal logic formulae are verified on the patient model, there is the need of a something enabling this task. We use formal verification environments, considering mathematical reasoning aimed to verify whether a model satisfies a set of requirements (i.e., the properties, in this case $\varphi$-coronal and $\psi$-axial respectively characterizing the prostate cancer in the coronal and in the axial slices). Among all the formal technique proposed from the scientific community, in this paper we use the model checking. The model checker accepts as input the formal model and the properties: basically, this technique evaluates the properties against the formal model, whether the model satisfies the property the model checker will output "true" (i.e., cancer), "false" (i.e., benign) otherwise. In this paper, the model checker implemented in the Concurrency Workbench of the New Century (CWB-NC) ${ }^{10}$ software is considered.

To demonstrate the effectiveness of the proposed method, we obtain a data-set composed of 80 MRIs ( 40 coronals and 40 axials) belonging to 40 patients. The MRIs were downloaded by authors from the picture archiving and communication system (PACS) of the "Centro Radiologico Potito"(https://www.centroradiologicopotito.it/), a radiological center in the city of Campobasso in Italy. The MRIs were acquired using a Philips Ingenia 1.5 T. The MRIs were in the digital imaging and communications in Medicine (DICOM) format and they are accompanied with the relative medical reports redact by radiologists with the cancer/benign indication. The patient dataset was obtained in accordance with relevant guidelines and regulations. All protocols were approved by the ethics committee/institutional review board of the Department of Medicine and Health "Vincenzo Tiberio", University of Molise, Campobasso, Italy. The informed consent was obtained from all subjects. Subject under 18 are not considered in the experimental evaluation.

\section{Results}

In this section we describe the experiment we performed to demonstrate the effectiveness of the proposed formal approach.

Figure 2 shows an example of (coronal and axial) benign and cancerous prostate MRIs belonging to the analysed data-set.

Table 2 shows the results for the formulae verification.

In Table 2 we add the $\checkmark$ symbol if the temporal logic properties are resulting true, Differently, if the patient model is labelled with false from the model checking software we add the $\boldsymbol{X}$ symbol. The $\varphi$-coronal column indicates the model checker output with the coronal slices, while the with $\psi$-axial the model checker output obtained with the axial slices. Moreover, the patient (medical report) column indicates, for each patient, the result of the radiologist diagnosis (i.e., cancer or benign), while 


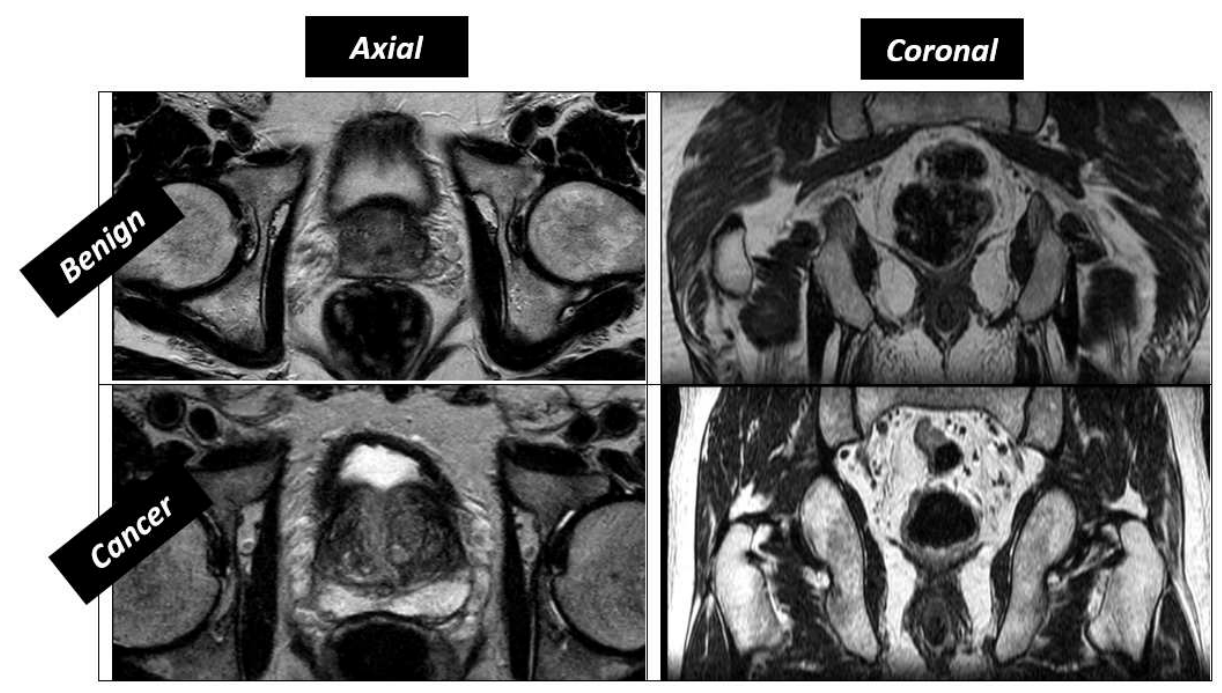

Figure 2. An example of benign and cancerous MRIs.

\begin{tabular}{|c|c|c|c|}
\hline $\begin{array}{ll}\text { Patient (medical report) } & \text { Formula verified on the model } \\
\end{array}$ & $\varphi$-coronal & $\psi$-axial & $\varphi \vee \psi($ software response) \\
\hline 1 (cancer) & $\sqrt{ }$ & $\sqrt{ }$ & $\sqrt{ }$ (cancer) \\
\hline 2 (benign) & $x$ & $x$ & $\boldsymbol{x}$ (benign) \\
\hline 3 (benign) & $x$ & $x$ & $\boldsymbol{x}$ (benign) \\
\hline 4 (cancer) & $x$ & $\sqrt{ }$ & $\sqrt{ }$ (cancer) \\
\hline 5 (benign) & $x$ & $x$ & $X$ (benign) \\
\hline 6 (benign) & $x$ & $x$ & $\boldsymbol{x}$ (benign) \\
\hline 7 (cancer) & $\checkmark$ & $\sqrt{ }$ & $x$ (benign) \\
\hline 8 (cancer) & $\sqrt{ }$ & $\sqrt{ }$ & $\checkmark$ (cancer) \\
\hline 9 (benign) & $x$ & $x$ & $\boldsymbol{X}$ (benign) \\
\hline 10 (cancer) & $\sqrt{ }$ & $\sqrt{ }$ & $\sqrt{ }$ (cancer) \\
\hline 11 (benign) & $x$ & $x$ & $\boldsymbol{x}$ (benign) \\
\hline 12 (cancer) & $\checkmark$ & $\sqrt{ }$ & $\checkmark$ (cancer) \\
\hline 13 (cancer) & $\checkmark$ & $\checkmark$ & $\checkmark$ (cancer) \\
\hline 14 (benign) & $x$ & $x$ & $x$ (benign) \\
\hline 15 (benign) & $x$ & $x$ & $\boldsymbol{x}$ (benign) \\
\hline 16 (benign) & $x$ & $x$ & $x$ (benign) \\
\hline 17 (benign) & $x$ & $x$ & $\boldsymbol{x}$ (benign) \\
\hline 18 (cancer) & $\checkmark$ & $\checkmark$ & $\checkmark$ (cancer) \\
\hline 19 (cancer) & $\checkmark$ & $\checkmark$ & $\checkmark$ (cancer) \\
\hline 20 (cancer) & $\checkmark$ & $\checkmark$ & $\checkmark$ (cancer) \\
\hline 21 (benign) & $x$ & $x$ & $\sqrt{ }$ (cancer) \\
\hline 22 (cancer) & $\checkmark$ & $\checkmark$ & $\checkmark$ (cancer) \\
\hline 23 (cancer) & $\checkmark$ & $\checkmark$ & $\checkmark$ (cancer) \\
\hline 24 (benign) & $x$ & $x$ & $\boldsymbol{x}$ (benign) \\
\hline 25 (cancer) & $\sqrt{ }$ & $\sqrt{ }$ & $\checkmark$ (cancer) \\
\hline 26 (cancer) & $\checkmark$ & $\checkmark$ & $\checkmark$ (cancer) \\
\hline 27 (benign) & $x$ & $x$ & $\boldsymbol{x}$ (benign) \\
\hline 28 (cancer) & $\checkmark$ & $\sqrt{ }$ & $\checkmark$ (cancer) \\
\hline 29 (cancer) & $\sqrt{ }$ & $\checkmark$ & $\checkmark$ (cancer) \\
\hline 30 (benign) & $x$ & $x$ & $\boldsymbol{x}$ (benign) \\
\hline 31 (benign) & $x$ & $x$ & $x$ (benign) \\
\hline 32 (benign) & $x$ & $x$ & $x$ (benign) \\
\hline 33 (benign) & $x$ & $x$ & $x$ (benign) \\
\hline 34 (benign) & $x$ & $x$ & $x$ (benign) \\
\hline 35 (cancer) & $\checkmark$ & $\sqrt{ }$ & $\sqrt{ }$ (cancer) \\
\hline 36 (benign) & $x$ & $x$ & $\boldsymbol{x}$ (benign) \\
\hline 37 (cancer) & $\checkmark$ & $x$ & $\sqrt{ }$ (cancer) \\
\hline 38 (cancer) & $\checkmark$ & $\sqrt{ }$ & $\checkmark$ (cancer) \\
\hline 39 (benign) & $x$ & $x$ & $\boldsymbol{x}$ (benign) \\
\hline 40 (cancer) & $\sqrt{ }$ & $\sqrt{ }$ & $\checkmark$ (cancer) \\
\hline
\end{tabular}

Table 2. Formulae verification.

the $\varphi$-coronal $\vee \psi$-axial indicated the software response produced by our developed method. Clearly, the models obtained by the coronal slices are verified with the $\varphi$-coronal formula, while the models obtained by the axial slices are verified using the 
$\psi$-axial one: the software response is $\varphi$-coronal $\vee \psi$-axial that is "true" (i.e., cancer) whether at least one formula between $\varphi$-coronal and $\psi$-axial is verified on the respective model (clearly whether $\varphi$-coronal and $\psi$-axial are "true" the software response will be "true"). It is sufficient to find a carcinogenic area in at least one of the two planes (axial/coronal).

From the results shown in Table 2, we highlight the importance of using the two planes (coronal and axial). In fact, for the patient \#4, the $\varphi$-coronal is "false", while the $\psi$-axial is "true", and for the patient \#37, the $\varphi$-coronal is "true", while the $\psi$-axial is "false". Therefore, for both the patients, our approach correctly outputs "true", i.e., cancer, coherently with the output radiologist, since we evaluated the disjunction of two formulae. Note that, if we had considered just one plan, we would have gotten two wrong results.

We consider four well-known metrics to evaluate the effectiveness of the model checking based approach, shown in Table 3. In reference ${ }^{11}$ there are more details available.

\begin{tabular}{cccc}
\hline \hline Sensitivity & Specificity & PPV & NPV \\
\hline 1 & 1 & 1 & 1 \\
\hline \hline
\end{tabular}

Table 3. Performance results.

The evaluation results in Table 3 show that model checking is very promising in prostate cancer detection; in fact a sensitivity and specificity equal to 1 are obtained.

An interesting aspect of the proposed method is represented by the counter-example, generated when a property fails to hold. The examination of this counter-example allows to understand why a property is not satisfied on a certain formal model. In this way it is possible to explain the reason why a certain property (in this case related to prostate cancer detection) is not satisfied on an MRI and to formulate and explanatory report. This is an interesting aspect, considering that in recent times research community is investigating about explainability ${ }^{12}$ (i.e., the process aimed to enable human users to develop understanding to be able to trust the model prediction ${ }^{13}$ ) of the prediction of medical decision systems, using machine learning techniques.

\section{Discussion}

In this section, state-of-the-art literature about the detection of prostate cancer is provided. We recall that, with respect to the methods proposed in the current literature, the main novelties introduces by the proposed method is the adoption of formal methods for prostate cancer detection exploiting radiomic feature from MRIs without the ROI. This represents one of the main novelties of the proposed method in fact, current literature present methods based on the ROI area to detect the cancer, this is symptomatic that these methods are not able to detect the cancerous area outside the marked ROI. For this reason our analysis is focused on the whole organ.

Researchers in ${ }^{14}$ investigate tissue images to detect prostate cancer. They propose a deep learning classifier, reaching an accuracy equal to 0.81 . The proposed method consider the distinctive mathematical rigor provided by formal methods, moreover we highlight that the proposed method do not require to the ROI for the cancer detection. Furthermore, we obtain better performances, as highlighted by the results obtained in the experimental analysis section.

Another deep learning architecture is evaluated in ${ }^{15}$ with the aim to detect prostate cancer. The obtain an accuracy equal to 0.70. Differently from authors in ${ }^{15}$ we exploit radiomics features to build from MRI slices through an automaton, reaching better performances.

Several methods ${ }^{16-19}$ consider supervised machine learning to build models to discriminate between bening and malignant prostate MRIs.

Authors in ${ }^{16}$ propose an invasive method, as a matter of fact they consider tissue image that can be obtained only from biopsy and then they classify these images for prostate cancer detection.

Bonekamp and colleagues ${ }^{20}$ demonstrated that radiomic features obtain comparable performances if compared with apparent diffusion coefficient features with regard to prostate lesions.

Authors in $^{21}$ discuss several examples of application in medical imaging involving artificial intelligence, by concluding that the interdisciplinary between machine learning and radiology can conduct to predictive analysis and precision medicine, where a therapy is developed ad-hoc for a specific patient.

Researchers in $^{22}$ demonstrate the radiomic features, mainly related to the second-order texture features for the detection of prostate canceres scored as PI-RADS 3 and 4, while authors in $^{23}$ are focused on the diagnostic performance of texture radiomic features for the detection of PI-RADS 3 lesions.

Recently, formal methods were applied from authors in ${ }^{11,24}$ for the prostate cancer Gleason score detection. Differently from following proposal, methods in ${ }^{11,24}$ require to pathologists to manually draw the ROI on the cancerous area to output the Gleason score. 
There are following novelty points with respect to these two methods: the adoption of formal methods, the analysis using MRIs without the ROI, the analysis of both coronal plane and axial plane and the better performances achieved.

To support radiologists and pathologists in the prostate cancer early detection, we propose an approach aimed to identify prostate cancer. Our analysis considers magnetic resonance images without the Region Of Interest (ROI) and exploits formal methods to model coronal and axial MRIs in terms of labeled transition systems.

The evaluation on a data-set gathered from a radiological center in the city of Campobasso (Italy) by showing that our approach is aimed to obtain a sensitivity and a specificity equal to 1 on a data-set composed from MRIs of 40 patients. We plan to extend our analysis to sagittal MRI slices. Moreover, we are interested to verify the generalization of our approach on the detection of other kind of cancers.

\section{References}

1. Center, M. M. et al. International variation in prostate cancer incidence and mortality rates. Eur. urology 61, 1079-1092 (2012).

2. Chen, W. et al. Cancer statistics in china, 2015. CA: a cancer journal for clinicians 66, 115-132 (2016).

3. La Manna, F. et al. Metastases in prostate cancer. Cold Spring Harb. perspectives medicine 9, a033688 (2019).

4. Turkbey, B. et al. Prostate imaging reporting and data system version 2.1: 2019 update of prostate imaging reporting and data system version 2. Eur. urology (2019).

5. Marshall, C. H. et al. Prevalence of dna repair gene mutations in localized prostate cancer according to clinical and pathologic features: association of gleason score and tumor stage. Prostate cancer prostatic diseases 22, 59 (2019).

6. Lorenz, J. W. et al. Serial t2-weighted magnetic resonance images acquired on a 1.5 tesla magnetic resonance linear accelerator reveal radiomic feature variation in organs at risk: An exploratory analysis of novel metrics of tissue response in prostate cancer. Cureus 11 (2019).

7. Van Griethuysen, J. J. et al. Computational radiomics system to decode the radiographic phenotype. Cancer research 77, e104-e107 (2017).

8. Dougherty, J., Kohavi, R. \& Sahami, M. Supervised and unsupervised discretization of continuous features. In Machine Learning Proceedings 1995, 194-202 (Elsevier, 1995).

9. Stirling, C. An introduction to modal and temporal logics for ccs. In Concurrency: Theory, Language, And Architecture, 2-20 (1989).

10. Cleaveland, R., Parrow, J. \& Steffen, B. The concurrency workbench. In International Conference on Computer Aided Verification, 24-37 (Springer, 1989).

11. Brunese, L., Mercaldo, F., Reginelli, A. \& Santone, A. Prostate gleason score detection and cancer treatment through real-time formal verification. IEEE Access 7, 186236-186246 (2019).

12. Parnas, D. L. Why engineers should not use artificial intelligence. INFOR: Inf. Syst. Oper. Res. 26, 234-246, DOI: 10.1080/03155986.1988.11732068 (1988).

13. Gilpin, L. H. et al. Explaining explanations: An overview of interpretability of machine learning. In 2018 IEEE 5th International Conference on data science and advanced analytics (DSAA), 80-89 (IEEE, 2018).

14. Kumar, N. et al. Convolutional neural networks for prostate cancer recurrence prediction. In Medical Imaging 2017: Digital Pathology, Orlando, Florida, United States, 11-16 February 2017, 101400H (2017).

15. Nagpal, K. et al. Development and validation of a deep learning algorithm for improving gleason scoring of prostate cancer. CoRR abs/1811.06497 (2018). 1811.06497.

16. Tabesh, A. et al. Multifeature prostate cancer diagnosis and gleason grading of histological images. IEEE transactions on medical imaging 26, 1366-1378 (2007).

17. Zhang, Y., Li, Q., Xin, Y. \& Lv, W. Differentiating prostate cancer from benign prostatic hyperplasia using psad based on machine learning: Single-center retrospective study in china. IEEE/ACM Transactions on Comput. Biol. Bioinforma. (2018).

18. Doyle, S., Madabhushi, A., Feldman, M. \& Tomaszeweski, J. A boosting cascade for automated detection of prostate cancer from digitized histology. In International Conference on Medical Image Computing and Computer-Assisted Intervention, 504-511 (Springer, 2006). 
19. Nguyen, T. H. et al. Automatic gleason grading of prostate cancer using slim and machine learning. In Quantitative Phase Imaging II, vol. 9718, 97180 Y (International Society for Optics and Photonics, 2016).

20. Bonekamp, D. et al. Radiomic machine learning for characterization of prostate lesions with mri: comparison to adc values. Radiology 289, 128-137 (2018).

21. Choy, G. et al. Current applications and future impact of machine learning in radiology. Radiology 288, 318-328 (2018).

22. Rudolph, M. M. et al. Diagnostic performance of pi-rads version 2.1 compared to version 2.0 for detection of peripheral and transition zone prostate cancer. Sci. reports 10, 1-10 (2020).

23. Giambelluca, D. et al. Pi-rads 3 lesions: role of prostate mri texture analysis in the identification of prostate cancer. Curr. problems diagnostic radiology 50, 175-185 (2021).

24. Brunese, L., Mercaldo, F., Reginelli, A. \& Santone, A. Formal methods for prostate cancer gleason score and treatment prediction using radiomic biomarkers. Magn. resonance imaging (2019). 
Figures

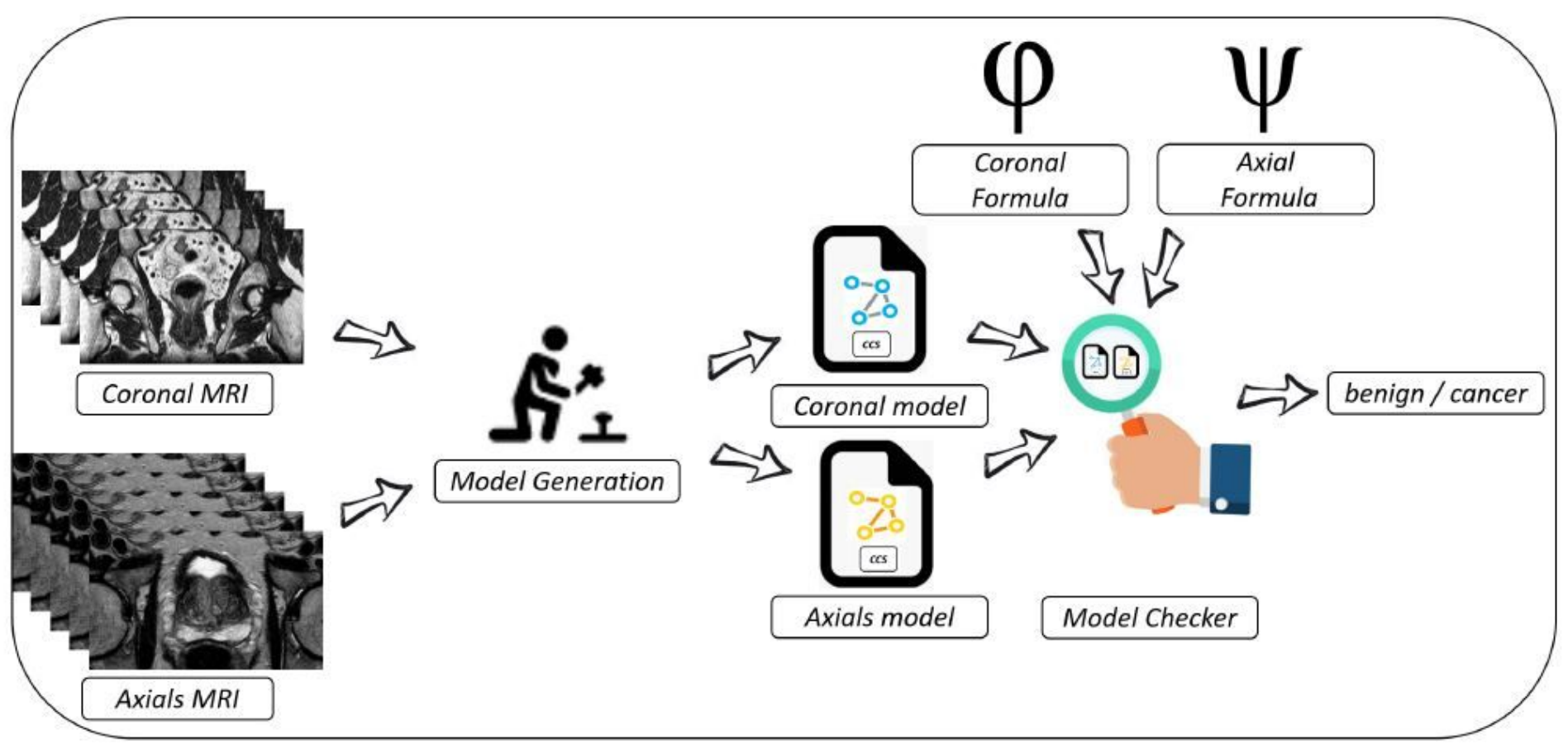

Figure 1

The method.

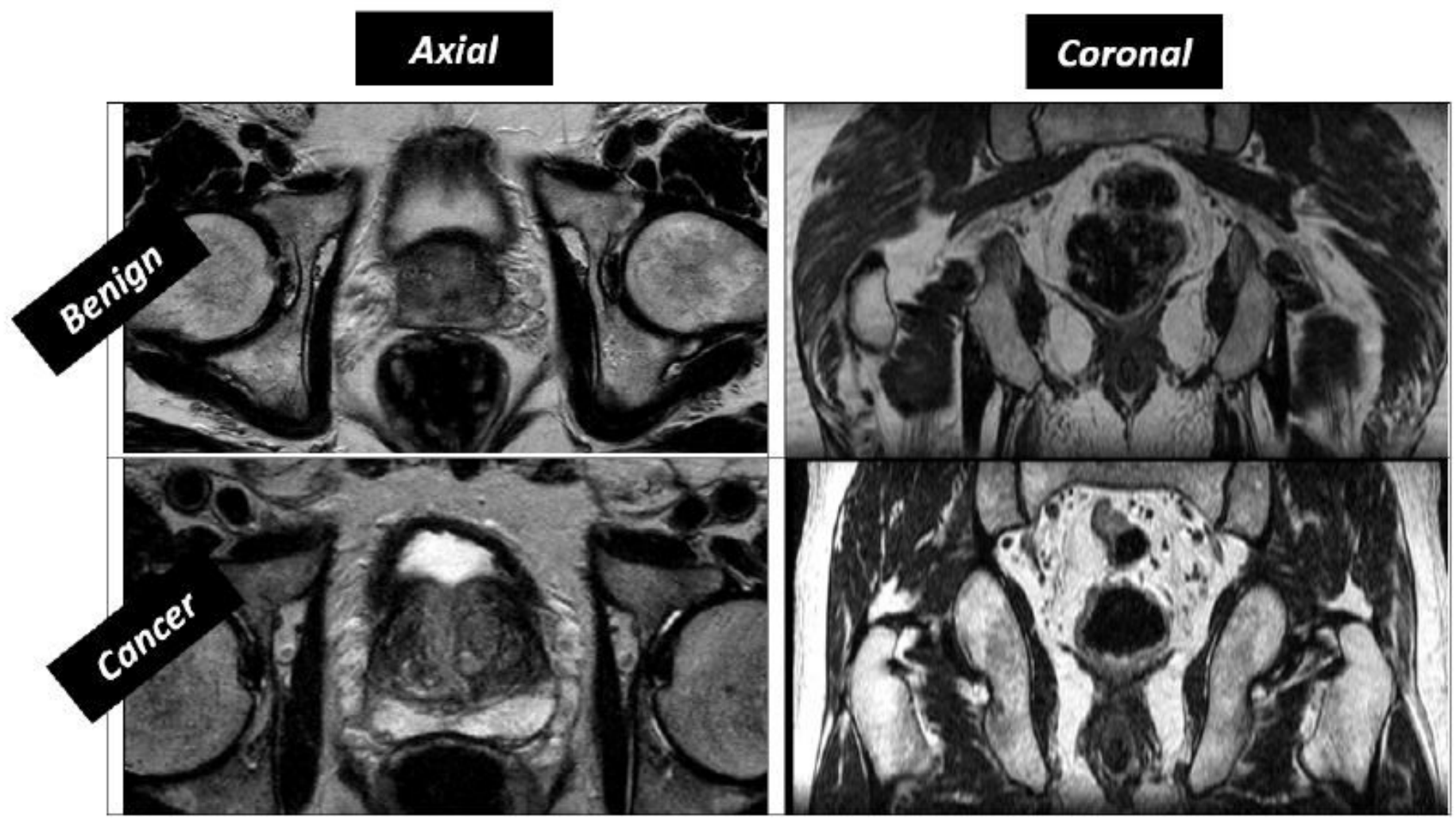


Figure 2

An example of benign and cancerous MRIs. 